\title{
Gearbox vibration signal amplitude and frequency modulation
}

\author{
Fakher Chaari $^{\mathrm{a}, *}$, Walter Bartelmus ${ }^{\mathrm{b}}$, Radoslaw Zimroz ${ }^{\mathrm{b}}$, Tahar Fakhfakh ${ }^{\mathrm{a}}$ and Mohamed Haddar ${ }^{\mathrm{a}}$ \\ ${ }^{a}$ Dynamics of Mechanical Systems Research Unit, National School of Engineers of Sfax, Sfax, Tunisia \\ ${ }^{\mathrm{b}}$ Diagnostics and Vibro-Acoustic Laboratory, Wroclaw University of Technology, Poland
}

Received 27 April 2010

Revised 19 May 2011

\begin{abstract}
Gearboxes usually run under fluctuating load conditions during service, however most of papers available in the literature describe models of gearboxes under stationary load conditions. Main task of published papers is fault modeling for their detection. Considering real situation from industry, the assumption of stationarity of load conditions cannot be longer kept. Vibration signals issued from monitoring in maintenance operations differ from mentioned models (due to load non-stationarity) and may be difficult to analyze which lead to erroneous diagnosis of the system. The objective of this paper is to study the influence of time varying load conditions on a gearbox dynamic behavior. To investigate this, a simple spur gear system without defects is modeled. It is subjected to a time varying load. The speed-torque characteristic of the driving motor is considered. The load variation induces speed variation, which causes a variation in the gearmesh stiffness period. Computer simulation shows deep amplitude modulations with sidebands that don't differ from those obtained when there is a defective tooth. In order to put in evidence the time varying load effects, Short Time Fourier Transform and then Smoothed Wigner-Ville distribution are used. Results show that the last one is well suited for the studied case.

The experimental validation presented at the end of the paper confirms the obtained results. Such results offer useful information when diagnosing gear transmissions by avoiding confusing conclusions from vibration signals.
\end{abstract}

Keywords: Gear transmission, varying load, gearmesh stiffness, amplitude modulation, frequency modulation, time frequency analysis

\section{Introduction}

Rotating machines are widely used in the manufacturing of industrial products. The gearbox, as a key rotating motion transmission component plays a critical role in the stable operation of rotating machinery and thus attracts considerable research interests especially in the condition monitoring and maintenance field. However, this monitoring must help detecting only the defects occurring in the gearbox and not the influence of non-deterioration sources of vibration. This is the case when the system is subjected to varying operating condition such as fluctuating load condition or torque level considered as the major source of contribution to the energy of the measured vibration signal. In an industrial process, many unexpected or uncertain sources can contribute to the fluctuation of load condition. For example, in the machining process, the variation of load applied on tooling system is transmitted back to the output shaft of a gearing system. As a consequence, considering only steady-state loads represent a very simplifying assumption in the modeling of gear systems. In fact, external load are often not constant and real machines are subjected to real operating conditions, which generally are represented by time varying loads. Gearbox vibration signals in this case may reveal time-varying signal to noise ratio, time varying frequency content, nonlinear

\footnotetext{
* Corresponding author: Fakher Chaari, Dynamics of Mechanical Systems Research Unit, National School of Engineers of Sfax, University of Sfax, BP 1173-3038, Sfax, Tunisia. Tel.: +216 98637156; Fax: +216 74 275595; E-mail: fakher.chaari@gmail.com.
} 
phenomena (links between sources of excitation and gearbox' response) etc... . To demonstrate all these statements, two possible approaches can be done: experimental work and model based work.

Bartelmus and Zimroz [1,2] showed that the identification of the varying external load is crucial to the evaluation of a planetary gearbox condition. Randall [3] states that the amplitude of a gearbox casing vibration, caused by the meshing of the gears, is modulated by the fluctuation of torque load. Stander [4] developed a simplified mathematical model to simulate the structural response and the instantaneous angular speed of a gear system under cyclic stationary and non-cyclic stationary load fluctuation. Baydar and Ball [5] indicate that spectral analysis cannot track the degradation in the condition of a gear under different constant load conditions. They employed the Instantaneous Power Spectrum to detect local faults on teeth in such conditions. Combet and Zimroz [6] proposed an original method for the estimation of the instantaneous speed fluctuation in a vibration signal measured on a system submitted to relatively heavy load and speed fluctuations.

Zhan et al. [7] proposed a novel technique for state detection of gearbox under varying load condition, which fits a time-varying autoregressive model to the gear motion residual signals applying a noise-adaptive filter.

All the previous papers provided diagnostic information under fluctuating loads and speeds. No rigorous mathematical modeling of the influence of varying loads on the gearmesh stiffness taking into account the torque-load characteristics of the driving motors was found in literature. This stiffness is time varying and is a key parameter in all gear transmissions and at the origin of the observed noise and vibrations [8-10]. Its variation is due to the fluctuation of the number of teeth in contact. In the non-stationary case, we have additional source that is time varying period of meshing due to variation of speed caused by variation of external load.

Starting from the positive experience expressed in cited papers [8-12], this paper will discuss the dynamic behavior of a spur gear transmission composed of a motor, gearbox and machine that work under time varying loading conditions. For simplicity, a single gear pair is used. The adopted model is a lumped parameters one with eight degrees of freedom which offers a global description of the dynamics of the transmission. The torque-speed characteristic of the driving motor is taken into account in order to compute the instantaneous rotational speed of the motor which is related to the load value. Dynamic response of the system is computed and the influence of the loading conditions is identified using Smoothed Wigner-Ville Distribution. The obtained results are compared with experimental results.

\section{Mathematical modeling of the transmission}

\subsection{Modeling of the spur gear system}

A one-stage spur gear model is considered [9]. It is divided into two rigid blocks as presented in Fig. 1. Each block has four degrees of freedom (two translations and two rotations). Pinion (12) with $Z_{1}$ teeth and gear (21) with $Z_{2}$ teeth are assumed to be rigid bodies and the shafts with torsional rigidity. Shafts are supported by rolling elements bearings modeled each one by two linear spring having constant stiffness. For the case of low contact ratio $(c<2)$, a fluctuation one pair-two pairs of teeth in contact is observed. This yields to a time varying gearmesh stiffness $K_{g}(t)$ acting along the line of action of the meshing teeth. This stiffness will be discussed later.

The expression of the displacement on the line of action is given by:

$$
\delta(t)=\left(x_{1}-x_{2}\right) \sin (\alpha)+\left(y_{1}-y_{2}\right) \cos (\alpha)+\theta_{12} r_{b 12}+\theta_{21} r_{b 21}
$$

$x_{i}$ and $y_{i}$ are the translations of block $i(i=1,2) . \theta_{i j}$ is the angular displacement of the wheel $j$ in block $i$ $(i, j=1,2) . r_{b 12}$ and $r_{b 21}$ are respectively the base radius of the pinion and the gear and $\alpha$ is the pressure angle. $q=\left\{x_{1}, y_{1}, \theta_{11}, \theta_{12}, x_{2}, y_{2}, \theta_{21}, \theta_{22}\right\}^{T}$ is the vector of degrees of freedom. A driving torque $T_{M}$ is applied on the inertia wheel 11 and the machine (22) exerts a load $T_{L}$ on the transmission. 


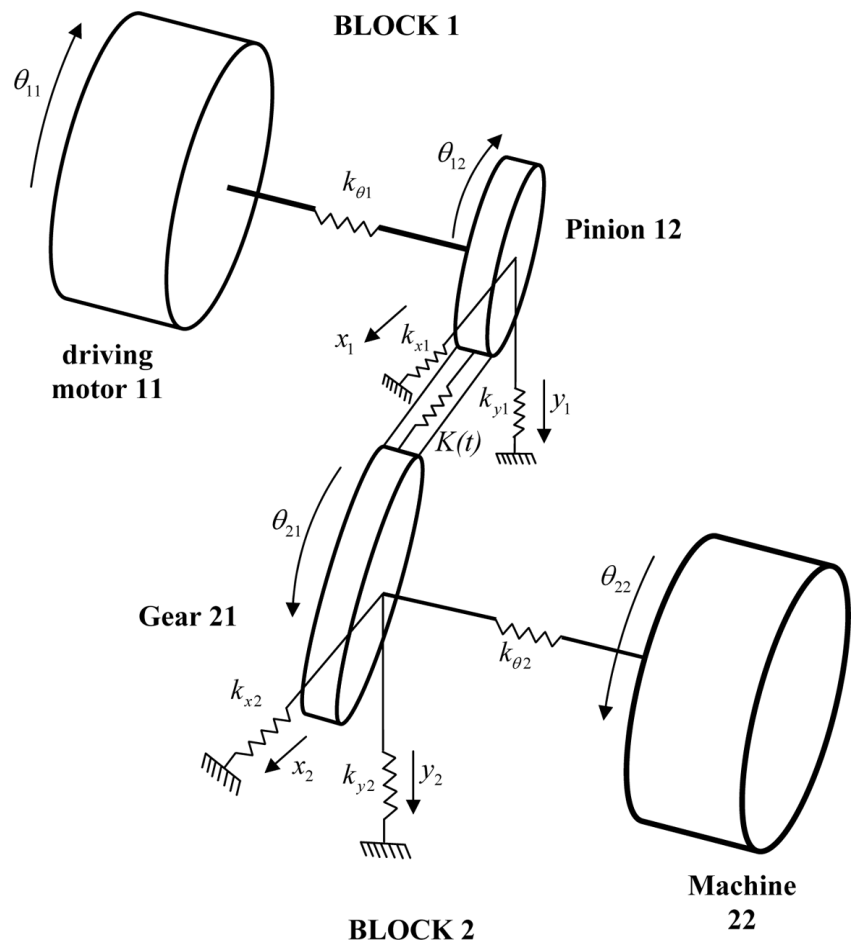

Fig. 1. Spur gear transmission modeling.

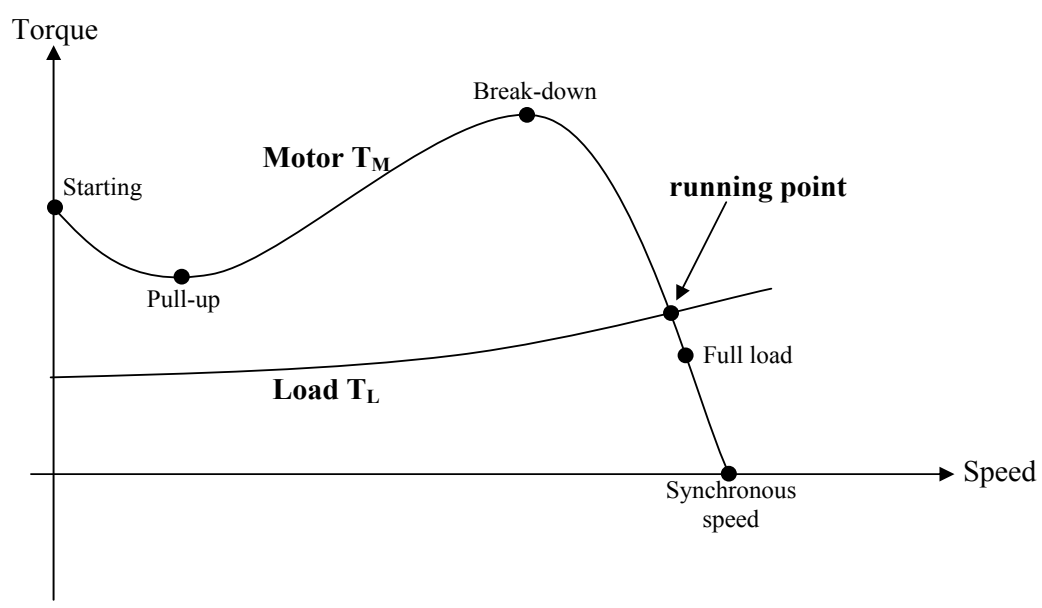

Fig. 2. Speed torque characteristics.

\subsection{Modeling of the torques}

The torque developed by a synchronous induction motor varies with its speed when it accelerates from full stop, to maximum operating speed [13].

Let us consider a motor connected to a load. The load includes a machine with a gearbox reducer. The motor torque and load characteristics are given in Fig. 2.

When energized, the motor torque $T_{M}$ must always exceed the torque absorbed by the load $T_{L}$ since the excess torque $T_{n e t}=T_{M}-T_{L}$ is necessary to accelerate the system of motor-plus-load until the system settles at a steady 


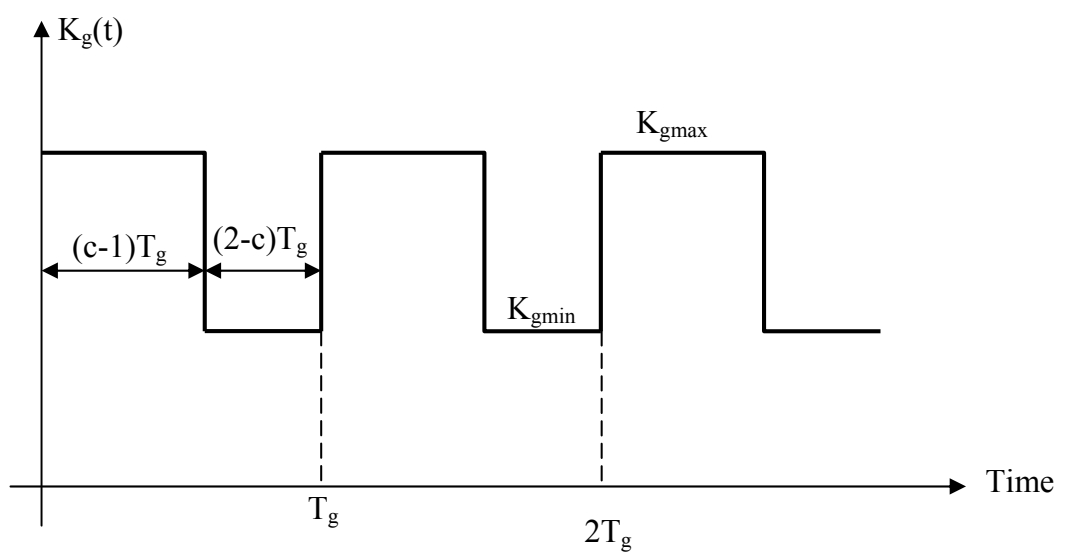

Fig. 3. Time varying evolution of $K_{g}(t)$ for a constant rotational speed $n_{r 1}$.

running speed $\mathrm{N}$ where torque equilibrium is reached, i.e. $T_{M}=T_{L}=T_{r}$, and the two characteristics intersect. The motor driving torque for a squirrel cage electric motor can be expressed by:

$$
T_{M}=\frac{T_{b}}{\left(1+\left(s_{b}-s\right)^{2}\left(\frac{a}{s}-b s^{2}\right)\right)}
$$

where $s_{b}$ and $T_{b}$ are the slip and torque at break-down (at maximum torque), $a$ and $b$ are all constant properties of the motor and $s$ is the proportional drop in speed given by:

$$
s=\left(N_{s}-n_{r}\right) / N s
$$

where $N_{s}$ is the synchronous speed and $n_{r}$ is the motor rotational speed. It can be noted that the greater the torque applied to the motor, the greater is the slip and the lower is the motor speed.

\subsection{Modeling of the time varying gearmesh stiffness}

Considering a rotational speed $n_{r 1}$ of the pinion which have $Z_{1}$ teeth, the gearmesh period (in seconds) is defined by:

$$
T_{g}=\frac{60}{n_{r 1} Z_{1}}
$$

When the rotational speed $n_{r 1}$ is constant, the time varying gearmesh stiffness $K_{g}(t)$ can be modeled by a periodic function (Fig. 3) so that the maximum values of stiffness $K_{\text {gmax }}$ corresponding to two pairs in contact are obtained from $\left(n T_{g}\right)$ to $(c-1)\left((n+1) T_{g}\right)$ and the minimum values $K_{g m i n}$ corresponding to one pair in contact are obtained from $(c-1)\left((n+1) T_{g}\right)$ to $\left((n+1) T_{g}\right)(n$ is integer) [8].

Now, if $n_{r 1}$ is fluctuating, $K_{g}(t)$ will be no longer periodic. The example given in Fig. 4 shows the decrease of the gearmesh stiffness period due to an overloading of the motor. In fact, an increase in the load will shift the running point (Fig. 2) and the motor have to develop, according to its characteristic, higher values of torque to overcome this overloading. Consequently, the rotational speed $n_{r 1}$ will decrease.

\subsection{Equation of motion of the system}

After defining the torques and gearmesh stiffness functions, the equation of motion of the system is obtained by applying Lagrange formulation and is given by:

$$
M \ddot{q}+C \dot{q}+K(t) q=T
$$

where $T=\left\{0,0, T_{M}, 0,0,0,0, T_{L}\right\}^{T}$ is the applied torques vector with $T_{L}=\frac{Z_{2}}{Z_{1}} T_{M} . M$ is a the mass matrix written as: 
Table 1

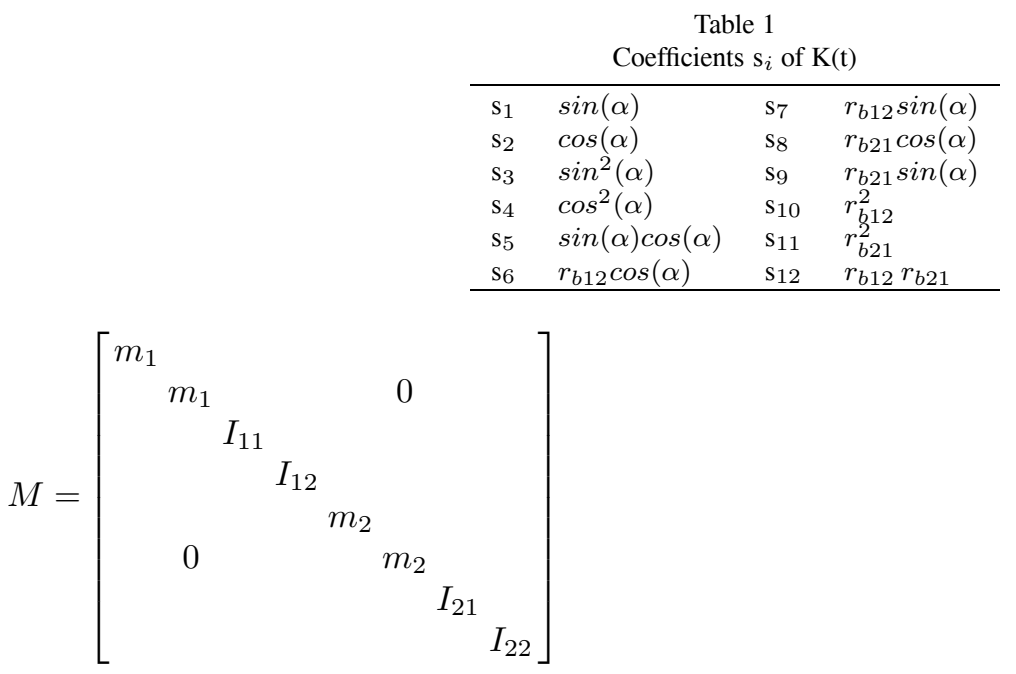

$m_{i}$ is the mass of block $i(i=1,2)$.

$I_{i j}$ is the mass moment of inertia of component $j$ in block $i,(i, j=1,2)$. For the driving motor and the load $I_{11}$ and $I_{22}$ are given by the manufacturer, whereas for pinion and gear the mass moment of inertia $I_{12}$ and $I_{21}$ are computed by considering these components as two disks. Then, $I_{12}$ and $I_{21}$ can be expressed by:

$$
\begin{aligned}
& I_{12}=\frac{m_{p}\left(d_{1} / 2\right)^{2}}{2} \\
& I_{21}=\frac{m_{g}\left(d_{2} / 2\right)^{2}}{2}
\end{aligned}
$$

Where $m_{p}$ and $m_{g}$ are the masses of pinion and gear, $d_{1}$ and $d_{2}$ are the pitch diameters.

$K(t)$ is written by [9]:

$$
K(t)=\left[\begin{array}{cccccccc}
s_{3} K_{g}(t)+k_{x 1} & s_{5} K_{g}(t) & 0 & s_{7} K_{g}(t) & -s_{3} K_{g}(t) & -s_{5} K_{g}(t) & 0 & s_{9} K_{g}(t) \\
s_{5} K_{g}(t) & s_{4} K_{g}(t)+k_{y 1} & 0 & s_{6} K_{g}(t) & -s_{5} K_{g}(t) & -s_{4} K_{g}(t) & 0 & s_{8} K_{g}(t) \\
0 & 0 & k_{\theta 1} & -k_{\theta 1} & 0 & 0 & 0 & 0 \\
s_{7} K_{g}(t) & s_{6} K_{g}(t) & -k_{\theta 1} & k_{\theta 1}+s_{10} K_{g}(t) & -s_{7} K_{g}(t) & -s_{6} K_{g}(t) & 0 & s_{12} K_{g}(t) \\
-s_{3} K_{g}(t) & -s_{5} K_{g}(t) & 0 & -s_{7} K_{g}(t) & s_{3} K_{g}(t)+k_{x 2} & s_{5} K_{g}(t) & 0 & -s_{9} K_{g}(t) \\
-s_{5} K_{g}(t) & -s_{4} K_{g}(t) & 0 & -s_{6} K_{g}(t) & s_{5} K_{g}(t) & s_{4} K_{g}(t)+k_{y 2} & 0 & s_{8} K_{g}(t) \\
0 & 0 & 0 & 0 & 0 & 0 & -k_{\theta 2} & -k_{\theta 2} \\
s_{9} K_{g}(t) & s_{8} K_{g}(t) & 0 & s_{12} K_{g}(t) & -s_{9} K_{g}(t) & s_{8} K_{g}(t) & -k_{\theta 2} & k_{\theta 2}+s_{11} K_{g}(t)
\end{array}\right](9)
$$

Where $k_{x i}, k_{y i}, k_{\theta i}(i=1,2)$ are respectively the bearings stiffnesses and torsional stiffnesses of shafts. The terms $s_{i}(i=1$ to12) are given in Table 1.

$K(t)$ can divide into a mean stiffness matrix $\bar{K}$ and a fluctuating one $k(t)$ :

$$
K(t)=\bar{K}+k(t)
$$

with

$$
\bar{K}=\left[\begin{array}{cccccccc}
k_{x 1} & 0 & 0 & 0 & 0 & 0 & 0 & 0 \\
0 & k_{y 1} & 0 & 0 & 0 & 0 & 0 & 0 \\
0 & 0 & k_{\theta 1} & -k_{\theta 1} & 0 & 0 & 0 & 0 \\
0 & 0 & -k_{\theta 1} & k_{\theta 1} & 0 & 0 & 0 & 0 \\
0 & 0 & 0 & 0 & k_{x 2} & 0 & 0 & 0 \\
0 & 0 & 0 & 0 & 0 & k_{y 2} & 0 & 0 \\
0 & 0 & 0 & 0 & 0 & 0 & -k_{\theta 2} & -k_{\theta 2} \\
0 & 0 & 0 & 0 & 0 & 0 & -k_{\theta 2} & k_{\theta 2}
\end{array}\right]
$$


Table 2

Motor characteristics

\begin{tabular}{lc}
\hline Motor type & ABB (Asea Brown Boveri) - MT 90L \\
\hline Electrical characteristics & 4 pole, $50 \mathrm{~Hz}, 3$ phase, $415 \mathrm{~V}$ \\
Output power $(\mathrm{kW})$ & 1.5 \\
Full load speed $(\mathrm{rpm})$ & 1420 \\
Synchronous speed $(\mathrm{rpm})$ & 1500 \\
Full load torque $\mathrm{T}_{f}(\mathrm{Nm})$ & 10 \\
Torque starting $\mathrm{T}_{s} / \mathrm{T}_{f}$ & 2.7 \\
Ratio breakdown $\mathrm{T}_{b} / \mathrm{T}_{f}$ & 3.2 \\
Rotor mass moment of inertia $\mathrm{I}_{11}\left(\mathrm{kgm}^{2}\right)$ & $4.3 \times 10^{-3}$ \\
Slip $\mathrm{s}_{b}$ & 0.315 \\
Motor constant a & 1.711 \\
Motor constant $\mathrm{b}$ & 1.316 \\
\hline
\end{tabular}

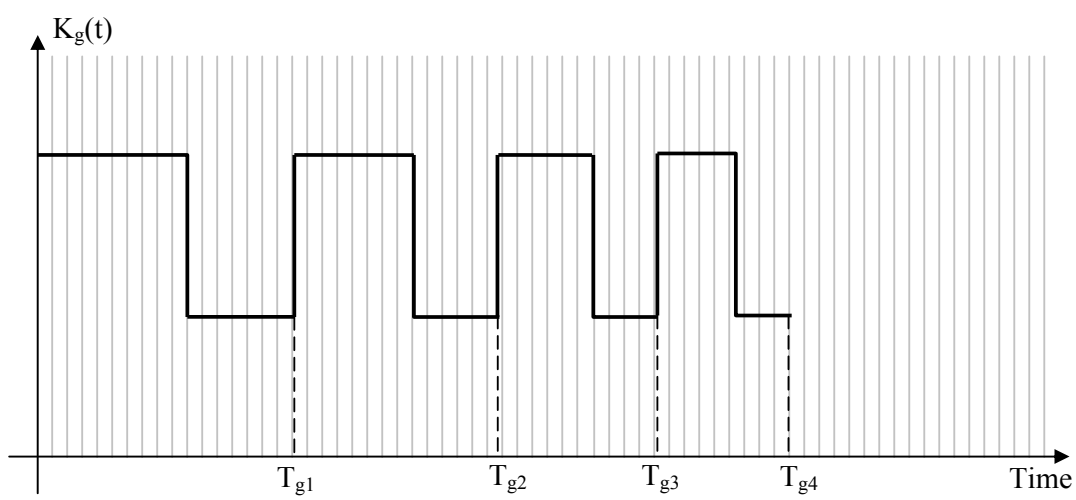

Fig. 4. Evolution of the gearmesh period of a decreasing rotational frequency $n_{r 1}$.

$$
k(t)=K_{g}(t)\left[\begin{array}{cccccccc}
s_{3} & s_{5} & 0 & s_{7} & -s_{3} & -s_{5} & 0 & s_{9} \\
s_{5} & s_{4} & 0 & s_{6} & -s_{5} & -s_{4} & 0 & s_{8} \\
0 & 0 & 0 & 0 & 0 & 0 & 0 & 0 \\
s_{7} & s_{6} & 0 & s_{10} & -s_{7} & -s_{6} & 0 & s_{12} \\
-s_{3} & -s_{5} & 0 & -s_{7} & s_{3} & s_{5} & 0 & -s_{9} \\
-s_{5} & -s_{4} & 0 & -s_{6} & s_{5} & s_{4} & 0 & s_{8} \\
0 & 0 & 0 & 0 & 0 & 0 & 0 & 0 \\
s_{9} & s_{8} & 0 & s_{12} & -s_{9} & s_{8} & 0 & s_{11}
\end{array}\right]
$$

$C$ is the proportional damping matrix expressed by [14]:

$$
C=0.05 M+10^{-6} \bar{K}
$$

The undamped eigenvalue problem derived from Eq. (5) by considering only the mean stiffness matrix $\bar{K}$ is:

$$
\left(-\omega_{i}^{2} M+\bar{K}\right) \phi_{i}=0
$$

Where $\phi_{i}$ is the eigenvector and $\omega_{i}$ the corresponding eigenfrequency.

\section{Numerical simulations}

\subsection{Dynamic response computation}

Let us consider a spur gear system driven by a squirrel cage motor. Its characteristics are given in Table 2 [13].

Figure 5 shows the speed-torque characteristic computed according to Eqs (2) and (3) and parameters given in Table 2. 
Table 3

Parameters of the spur gear transmission

\begin{tabular}{|c|c|c|}
\hline & Pinion & Wheel \\
\hline Teeth numbers & 20 & 40 \\
\hline Mass $(\mathrm{kg})$ & 0.6 & 1.5 \\
\hline Mass moment of inertia $\left(\mathrm{kgm}^{2}\right)$ & $2.7 \times 10^{-4}$ & 0.0027 \\
\hline Full load torque (N.m) & 10 & -20 \\
\hline Rotation speed (rpm) & 3000 & 1500 \\
\hline Modulus (mm) & \multicolumn{2}{|c|}{3} \\
\hline Primitive diameter (mm) & 60 & 120 \\
\hline Base circle diameter $(\mathrm{mm})$ & 56.38 & 112.76 \\
\hline Material & \multicolumn{2}{|c|}{ 42CrMo4 } \\
\hline Density $\left(\mathrm{kg} / \mathrm{m}^{3}\right)$ & \multicolumn{2}{|c|}{$\rho=7860$} \\
\hline Poisson ratio & \multicolumn{2}{|c|}{0.33} \\
\hline Pressure angle & \multicolumn{2}{|c|}{$\alpha=20^{\circ}$} \\
\hline Teeth width (mm) & \multicolumn{2}{|c|}{23} \\
\hline Contact ratio & \multirow{4}{*}{\multicolumn{2}{|c|}{$\begin{array}{c}c=1.6 \\
\mathrm{k}_{x 1}=\mathrm{k}_{y 1}=\mathrm{k}_{x 2}=\mathrm{k}_{y 2}=10^{8} \\
k_{\theta 1}=k_{\theta 2}=10^{5} \\
\overline{K_{g}}=2 \times 10^{8}\end{array}$}} \\
\hline Bearing stiffness (N/m) & & \\
\hline Torsional stiffness ( $\mathrm{N} \mathrm{rd} / \mathrm{m})$ & & \\
\hline Mean gearmesh stiffness $(\mathrm{N} / \mathrm{m})$ & & \\
\hline
\end{tabular}

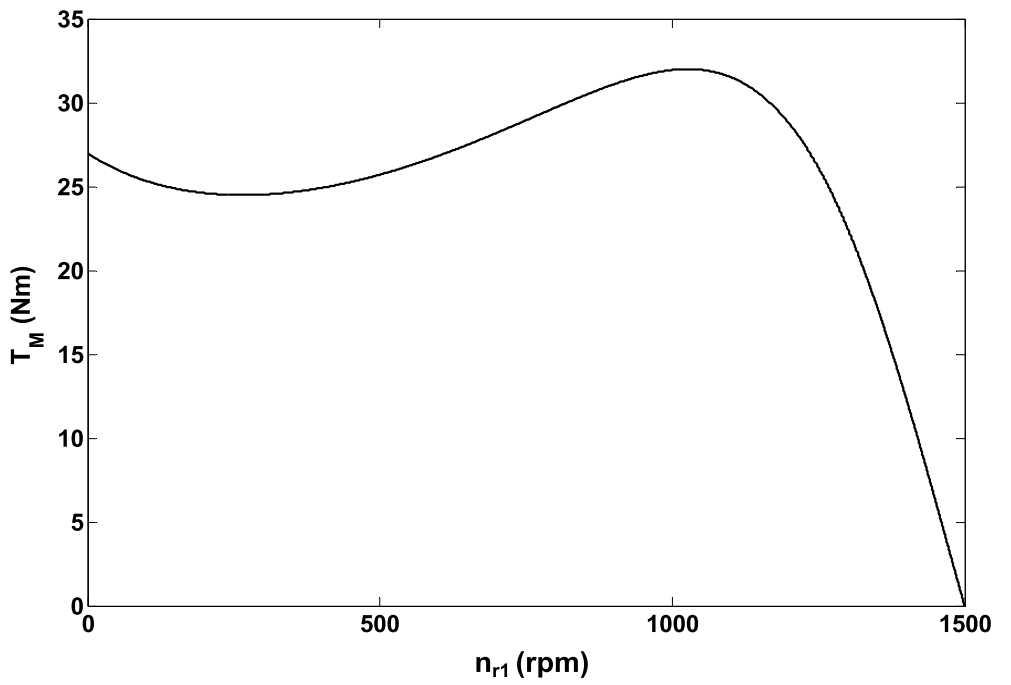

Fig. 5. Speed-Torque characteristic of the motor.

The parameters of the spur gear transmission are given in Table 3. The mass of the blocks I and II are $m_{1}=$ $1.8 \mathrm{~kg}$ and $m_{2}=2.5 \mathrm{~kg}$. The mass moment of inertia of the driving motor and the machine are $I_{11}=0.0043 \mathrm{kgm}^{2}$ and $I_{22}=0.0045 \mathrm{kgm}^{2}$.

For the studied system with 8 degrees of freedom, the eigenfrequencies in $\mathrm{Hz}$ are as follows: [0 - $696-795-$ 1146 - 1186 - 1399 - 1756 - 5203].

Let us consider that the load on machine (22) fluctuating in a saw-tooth shape with a frequency $f_{L}=15 \mathrm{~Hz}$ rising from 10 to $31.5 \mathrm{Nm}$ as presented in Fig. 6.

In order to overcome the periodic increase in load, the rotational speed of the motor will decrease following the characteristic given in Fig. 5. Its fluctuation is presented in Fig. 7.

A varying rotational speed will lead also a varying gearmesh frequency. The mean value of the motor rotational speed is $n_{r 1 m}=1310 \mathrm{rpm}$ which corresponds to a mean gearmesh frequency and period of $f_{g m}=436 \mathrm{~Hz}$ and $T_{g m}=0.0023 \mathrm{~s}$. The gearmesh stiffness function and its spectrum are given in Fig. 8, they are presented with non-dimensioned time and frequency axis.

From literature [8,9] the spectrum of a gear transmission submitted to a constant load is dominated only by the gearmesh frequency and its harmonics. However in the simulated case with time varying load, a family of sidebands 


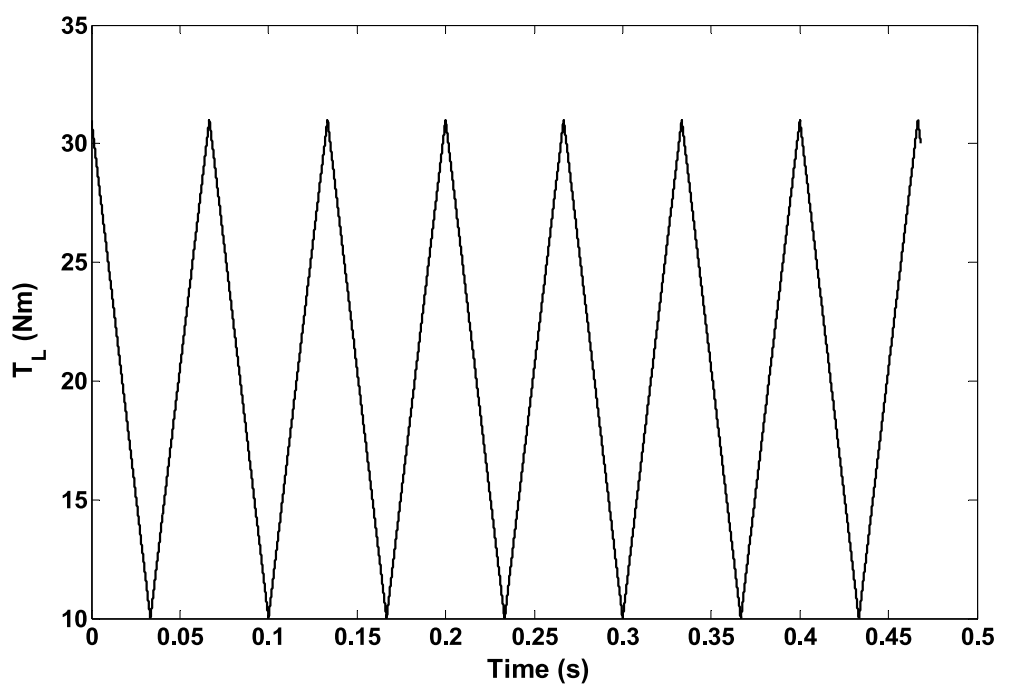

Fig. 6. Evolution of the load applied on the inertia wheel 22.

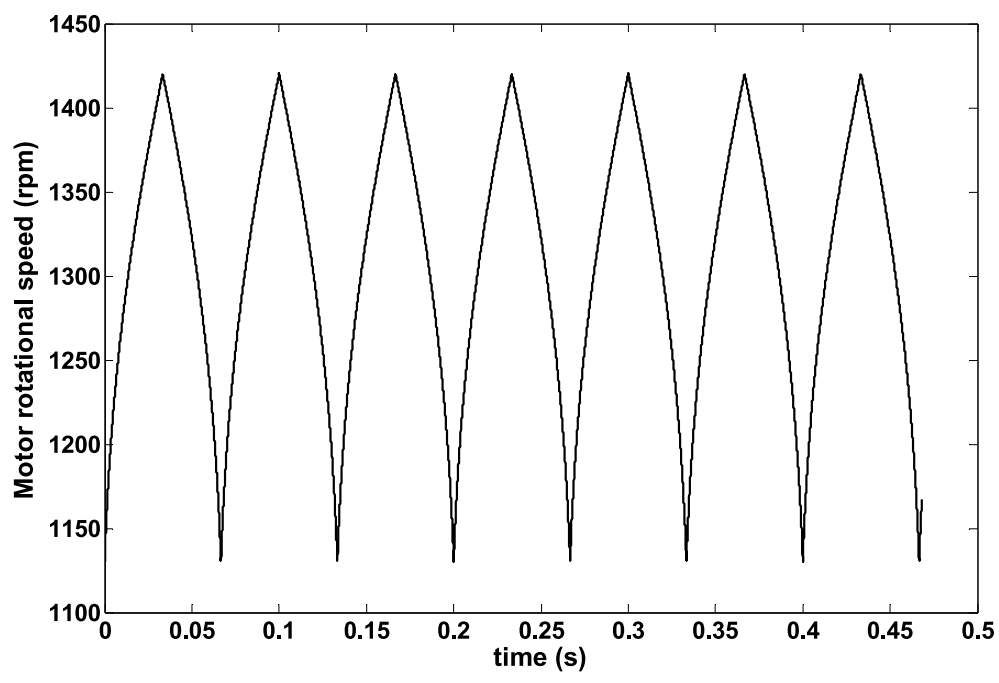

Fig. 7. Variation of the motor rotational speed.

appeared around the gearmesh frequency and its harmonics induced by the non uniformity of the gearmesh period (Fig. 8a) and this can be thought to be a frequency modulation of the gearmesh stiffness.

This gearmesh stiffness is inserted in the equation of motion (5) and the dynamic response of the system is computed using the implicit Newmark algorithm. At each time instant the solution is calculated taking into account the instantaneous value of $K_{g}(t)$. Another case with constant load condition is considered with a periodic function of $K_{g}(t)$ with frequency $f_{g m}=436 \mathrm{~Hz}$. Figures 9 and 10 show the time evolution and spectra of the acceleration on the pinion and wheel bearings for the constant load and time varying load conditions.

The dynamic response issued from the constant loading case (Figs 8-a and 9-a) is dominated by the harmonics of the mean gearmesh frequency especially $3 \times f_{g m}$ and $4 \times f_{g m}$. But when looking to time varying loading case, it is clearly noticed the frequency modulation effects characterized by deep amplitude modulation in the time responses and multiplicity of sidebands especially around the same harmonics of $f_{g m}$ close to the system eigenfrequencies. This result is obviously caused by the variation of the rotational speed of the system as a result of the load variation which shows a wide difference between its minimum and maximum values. Looking to these responses and ignoring the load fluctuation, one can conclude otherwise. For non-experts it may be considered as a 

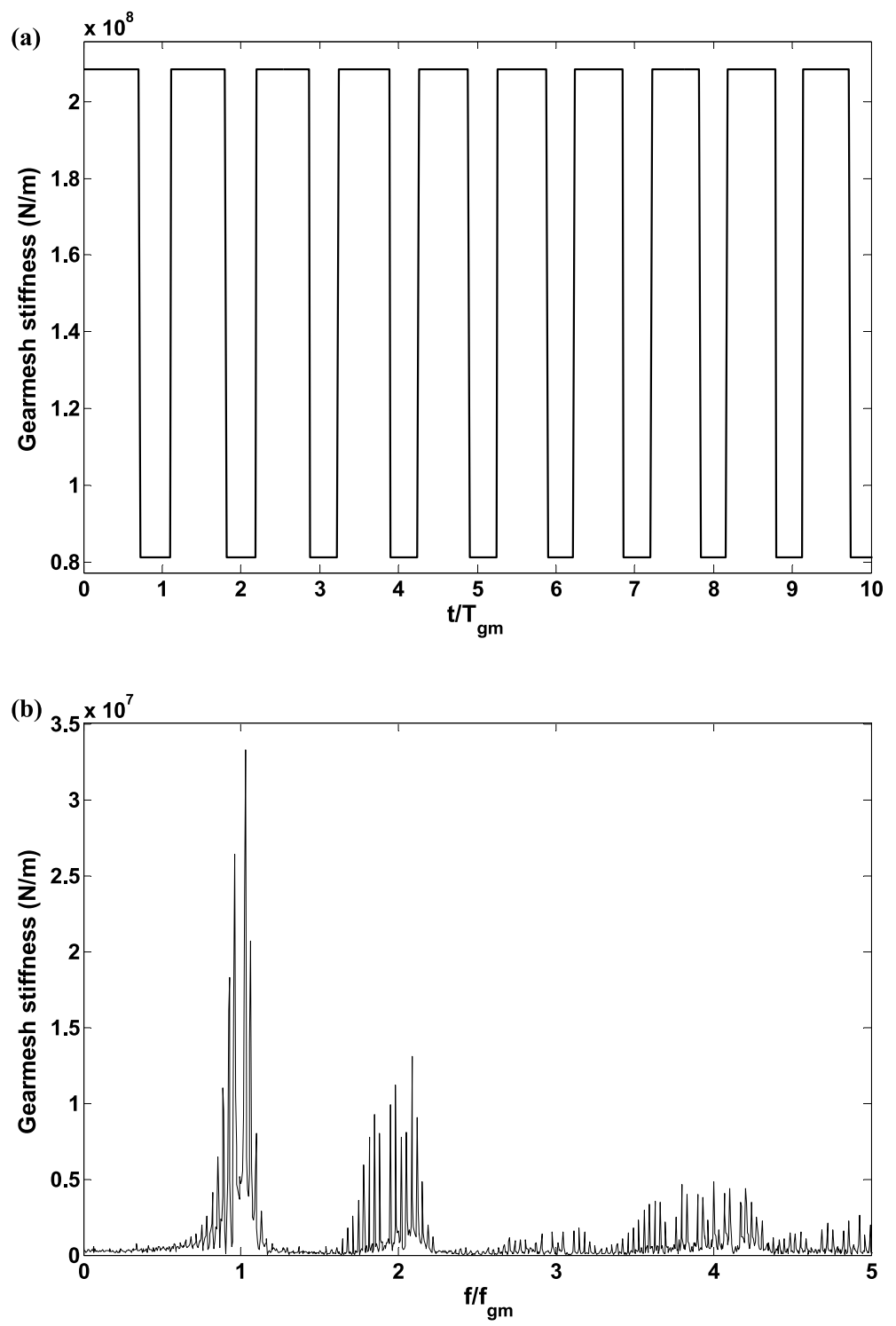

Fig. 8. (a) Gearmesh stiffness function (b) Spectrum of the variable part of the gearmesh stiffness function.

faulty gearbox, which is obviously not true in this case. In fact, similar results can be obtained if local faults such as tooth root crack, tooth breakage or a spall on a tooth flank occur $[3,8,9]$, with amplitude modulation of the gearmesh function by these defects. On the other hand, defective bearings can lead also to the same dynamic behavior [15]. In conclusion, separated time and frequency analysis for the studied case may mislead to wrong diagnosis of the spur gear transmission.

\subsection{Short time fourier transform analysis}

Time-frequency analysis is a three-dimensional time, frequency and amplitude representation of a signal, which is inherently suited to indicate transient events in a signal. The short-time Fourier transform (STFT) is a Fourier-related transform used to determine the sinusoidal frequency and phase content of local sections of a signal as it changes over time. Simply, in the continuous-time case, the function to be transformed is multiplied by a window function which is nonzero for only a short period of time. The Fourier transform (a one-dimensional function) of the resulting 

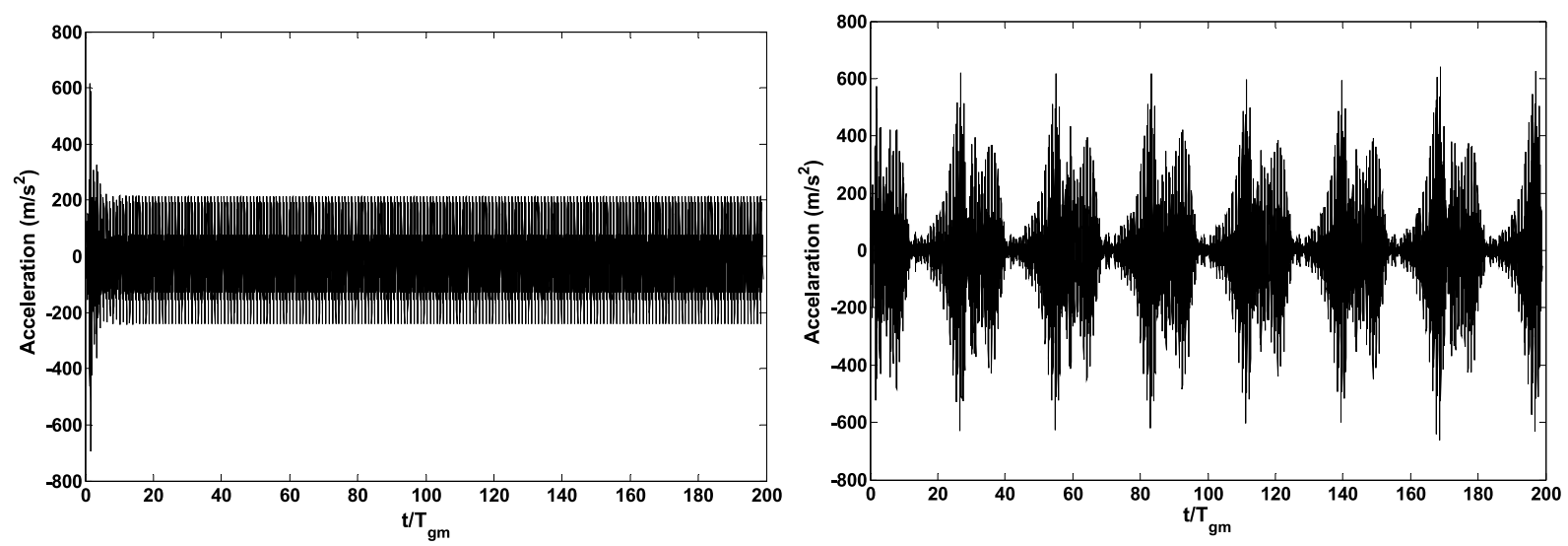

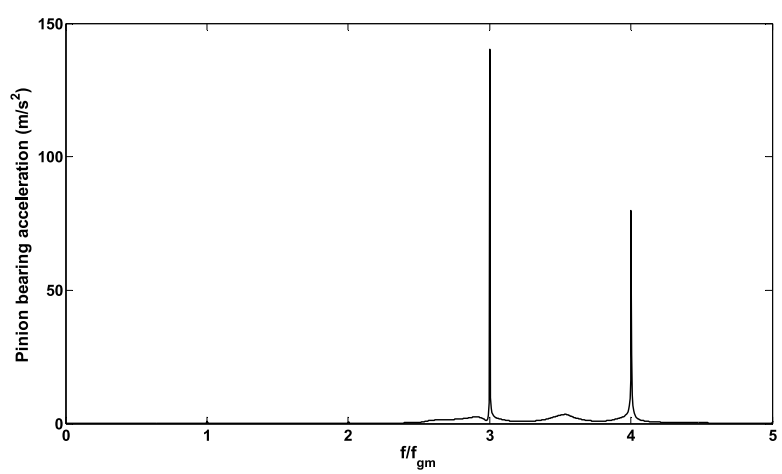

(a)

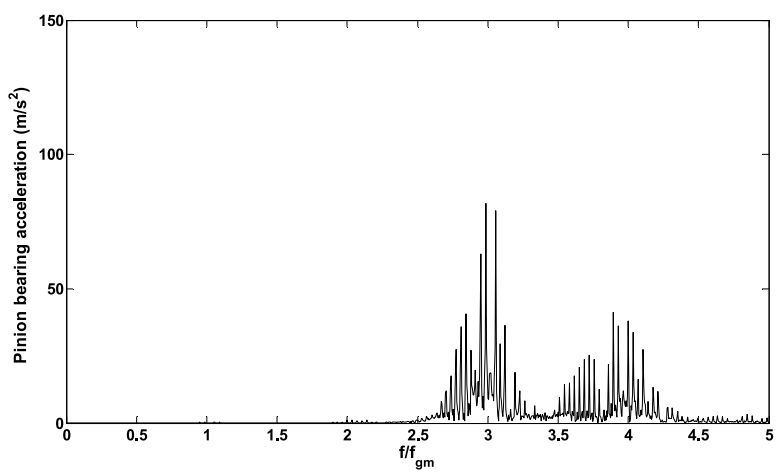

(b)

Fig. 9. Time evolution and spectra of the acceleration of the pinion bearing for constant load case (a) and time varying load case (b).

signal is taken as the window is sliding along the time axis, resulting in a two-dimensional representation of the signal [16]. Mathematically, this is written for a time signal $x(t)$ as:

$$
\operatorname{STFT}(t, \omega)=\int_{-\infty}^{+\infty} x(t) w(t-\tau) e^{-j \omega t} d t
$$

where $w(t)$ is the window function, commonly a Hann window or Gaussian "hill" centered around zero.

Figure 11 represents the STFT of the acceleration on the pinion bearing with a constant applied load corresponding to the mean value of $T_{L}(20.5 \mathrm{Nm})$ and Fig. 12 shows the STFT of the same acceleration but with the time varying applied load as given in Fig. 6.

When comparing theses two figures, the influence of the time varying load can be detected but with a limited resolution.

\subsection{Wigner-Ville time-frequency analysis}

One of the drawbacks of the STFT is that it has a fixed resolution. The width of the windowing function relates to how the signal is represented - it determines whether there is good frequency resolution (frequency components close together can be separated) or good time resolution (the time at which frequencies change). A wide window gives better frequency resolution but poor time resolution. A narrower window gives good time resolution but poor frequency resolution. These are called narrowband and wideband transforms, respectively. An alternative to STFT is the Wigner Ville distribution. This distribution and its various permutations have been used by various authors [16, 17] to detect the presence of local gear faults by means of vibration signal analysis. The Wigner distribution is derived by generalizing the relationship between the power spectrum and the autocorrelation function for non-stationary 

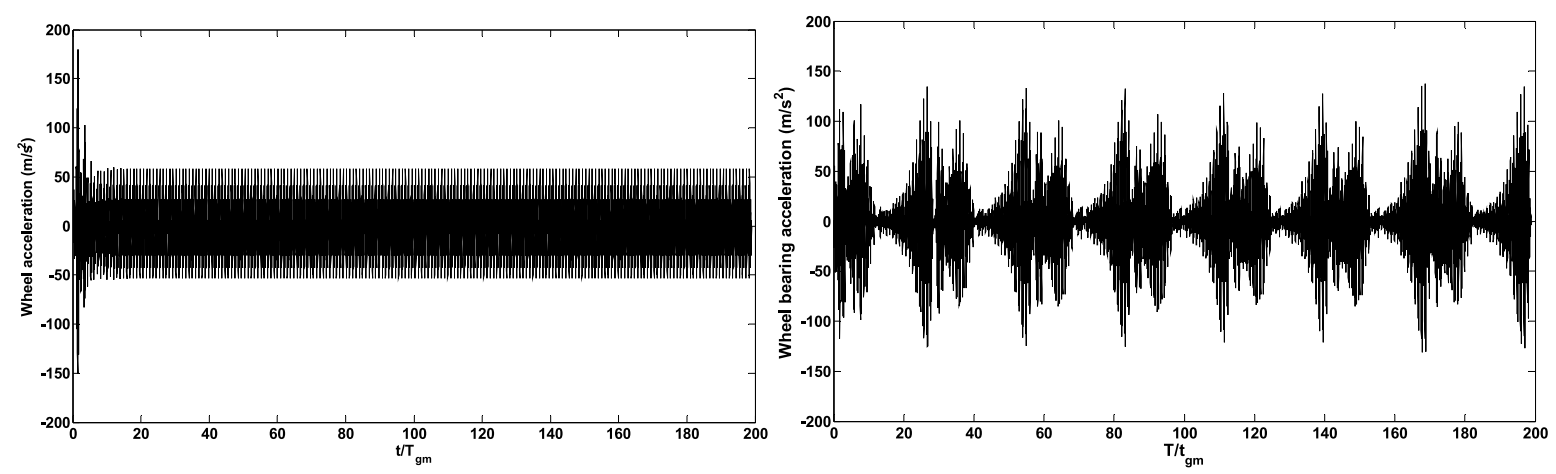

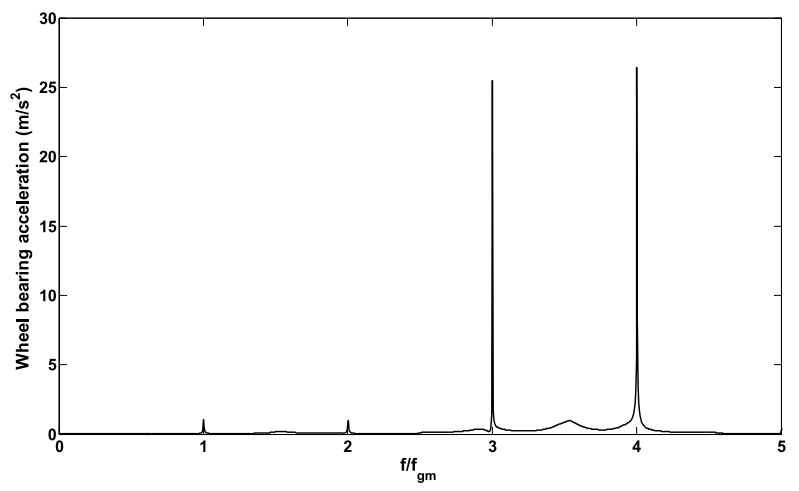

(a)

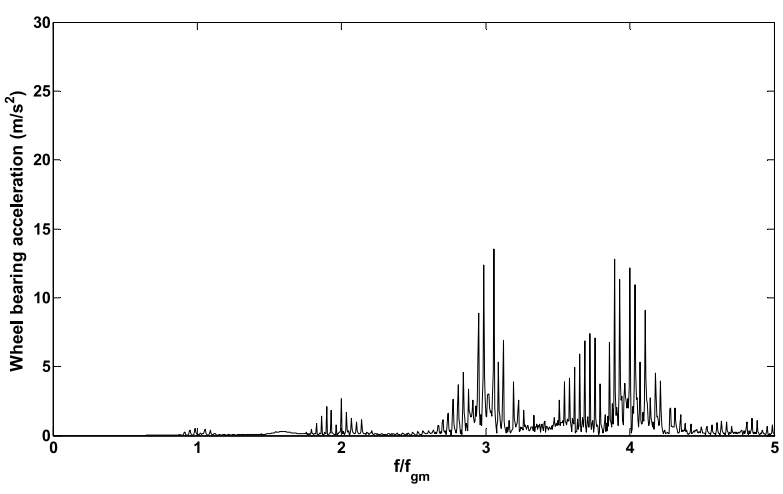

(b)

Fig. 10. Time evolution and spectra of the acceleration of the wheel bearing for constant load case (a) and time varying load case (b).

time-variant processes. The amplitude of the negative frequencies in the frequency domain is zero for an analytical signal. Therefore, the application of the Wigner distribution to the analytical signal will account for all frequencies. The application is referred to as the Wigner-Ville distribution. A frequency-smoothing window is applied to this distribution in order to attenuate the interference and cross-terms that are its inherent property of the distribution. This is performed by Smoothed Wigner-Ville Distribution (SWVD) [17]. It is defined for a time signal $s(t)$ by:

$$
S W V D_{x}(t, \omega)=\int_{-\infty}^{+\infty} h(\tau) s(t+\tau / 2) s^{*}(t-\tau / 2) e^{-j \omega t} d \tau
$$

where $t$ denotes the time, $\omega$ the frequency, $\tau$ the time delay and $h(\tau)$ the frequency-smoothing window. ${ }^{*}$ denotes complex conjugate. $s(t)$ an analytical signal obtained by:

$$
s(t)=x(t)+j H((x(t)))
$$

where $H((x(t)))$ is the Hilbert transform of $x(t)$.

Figure 13 represents the SWVD of the acceleration on the pinion bearing with a constant applied load corresponding to the mean value of $T_{L}(20.5 \mathrm{Nm})$ and Fig. 14 shows the SWVD of the same acceleration but with the time varying applied load given in Fig. 6.

When comparing Figs 13 and 14, it can be noticed a flat evolution of the mean gearmesh frequency $\left(f_{g m}\right)$ harmonics for the constant load case, whereas, sawtooth shapes of these harmonics are observed for the varying load case similar to that of the load variation signal with the same periodicity $1 / f_{L}=0.067 \mathrm{~s}$. Figure 15 shows details of Fig. 14 around $3 \times f_{g m}$.

It is well noticed a periodicity of the gearmesh frequency variation from a minimum value (corresponding to the maximum applied load) to a maximum value (corresponding to the minimum applied load). The increase of 


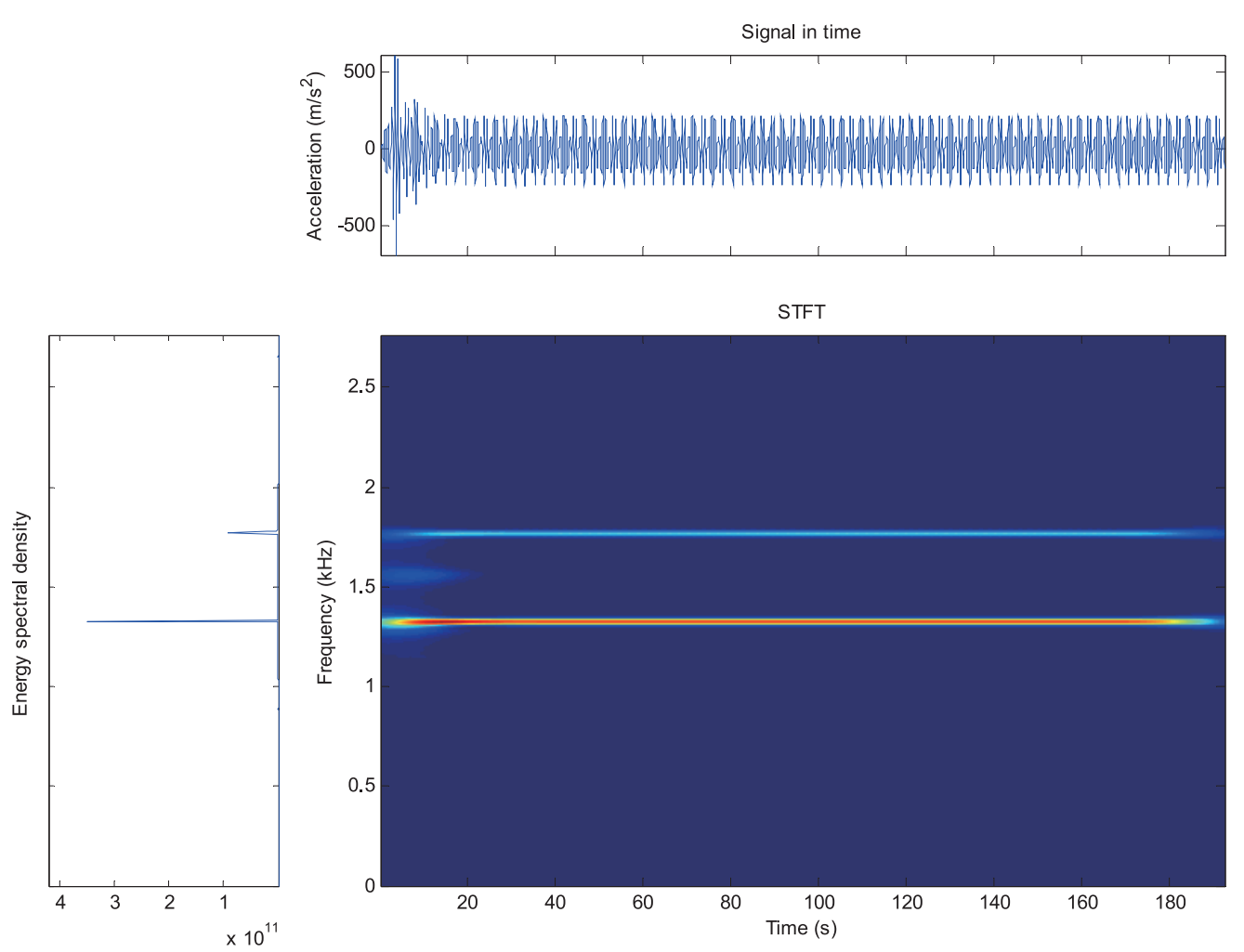

Fig. 11. STFT of the pinion bearing acceleration for a constant applied load.

vibration amplitude can also be detected for higher load values by the darkness observed in the charts. So, it can be concluded from the SWVD analysis that the origin of the sidebands is the not a gear defect but simply a load variation that is the responsible for the frequency modulation of the gearmesh stiffness function. This can clearly state about the efficiency of the SWVD in identifying the presence of fluctuating load and its identification.

\subsection{Experimental validation}

To validate the obtained results with real object measurements, the case of a mining machine (MM) is considered [18-20]. The main component of this machine is the driving unit (DU) consisting in a motor, a coupling, a multistage gearbox. At the output of the DU different types of driven machines (conveyor, bucket wheel etc...) may be used. This mechanical system generates complex vibration signals and moreover they influence each other. Additionally, MM work under time-varying, non-stationary operations that means time varying properties of signals (frequency structure, amplitudes, nonlinear phenomena etc.). Figure 16-a show a view of a bucket wheel during operation. The model of an external load caused by ground varying property, when a bucket wheel is used for digging, is given in Fig. 16-b. This causes a variation of the rotational speed of the electric driving motor as given in Fig. 16-c.

The authors noticed that periodic (cyclic) variation of external load causes amplitude modulation whereas speed variation (associated with load variation) causes frequency modulation $[1,20]$.

Examples presented on Fig. 17 shows that both phenomena exist in the system, and range of variation (influence to vibration signal) is significant.

Figure 17a presents raw vibration signal pre-filtered around one of mesh harmonic. Low-frequency envelope related to load variation is added. It should be noted that extraction of low-frequency envelope is not easy due to spectral overlapping with low speed shafts in investigated gearbox (load variation and shaft rotation have very similar cycle length). 

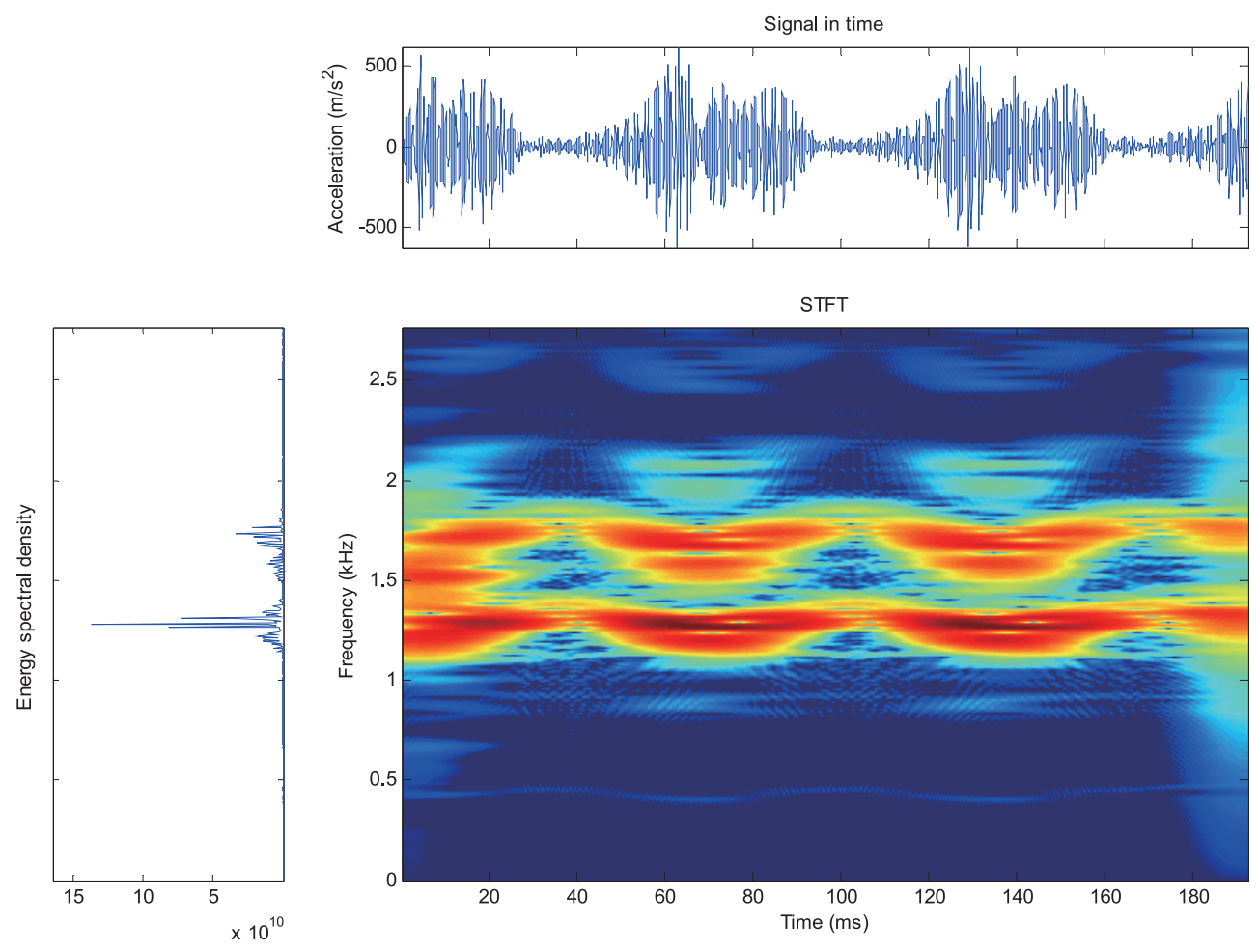

Fig. 12. STFT of the pinion bearing acceleration for a time varying applied load.

Figure 17b shows results of instantaneous (mesh) frequency estimation time in frequency domain (STFT) by a novel procedure described in [21].

It is worth to notice a difference of sawtooth shape. For low speed envelope (influence of torque) it is slowly increasing and quickly decreasing, however, for speed profile it is quickly increasing and slowly decreasing. This phenomenon is strictly related with mentioned speed torque characteristic (Fig. 2).

If frequency and amplitude modulations exist in the same time it makes spectral content complicated and difficult to analyze, even in time frequency plane. Most of frequency tracking techniques is sensitive to time varying signal to noise ratio, which appears due to amplitude modulation (Fig. 18).

The application of the STFT to accelerations measured on DU bearings is shown in Fig. 19.

As it was mentioned, one may identify both variation of frequency ( 3 harmonics are presented) and amplitudes (it is clearly seen for harmonic varying around $2600 \mathrm{~Hz}$ ).

It is noticed a similar evolution to that obtained by simulation (Figs 12 and 14) with periodic variation of the gearmesh frequency harmonics induced by DU speed variation.

Looking on the Figs 17 and 19 one may be conscious why mechanical signature of gearbox for time varying conditions is so similar to signature from faulty gearbox under stationary load. It is much easier understand data and recognize actual condition of the gearbox if operating condition are identified and their influence to vibration properly interpreted. Combining modeling and experimental work, i.e. model response and real signals analysis one may avoid mistakes during diagnosis of gearboxes.

\section{Conclusion}

In this paper, a problem of gearbox diagnosis under non-stationary operating conditions is considered.

It has been found that during time-varying load-speed conditions vibration signature of healthy gearbox may be identified as symptom of bad condition. 

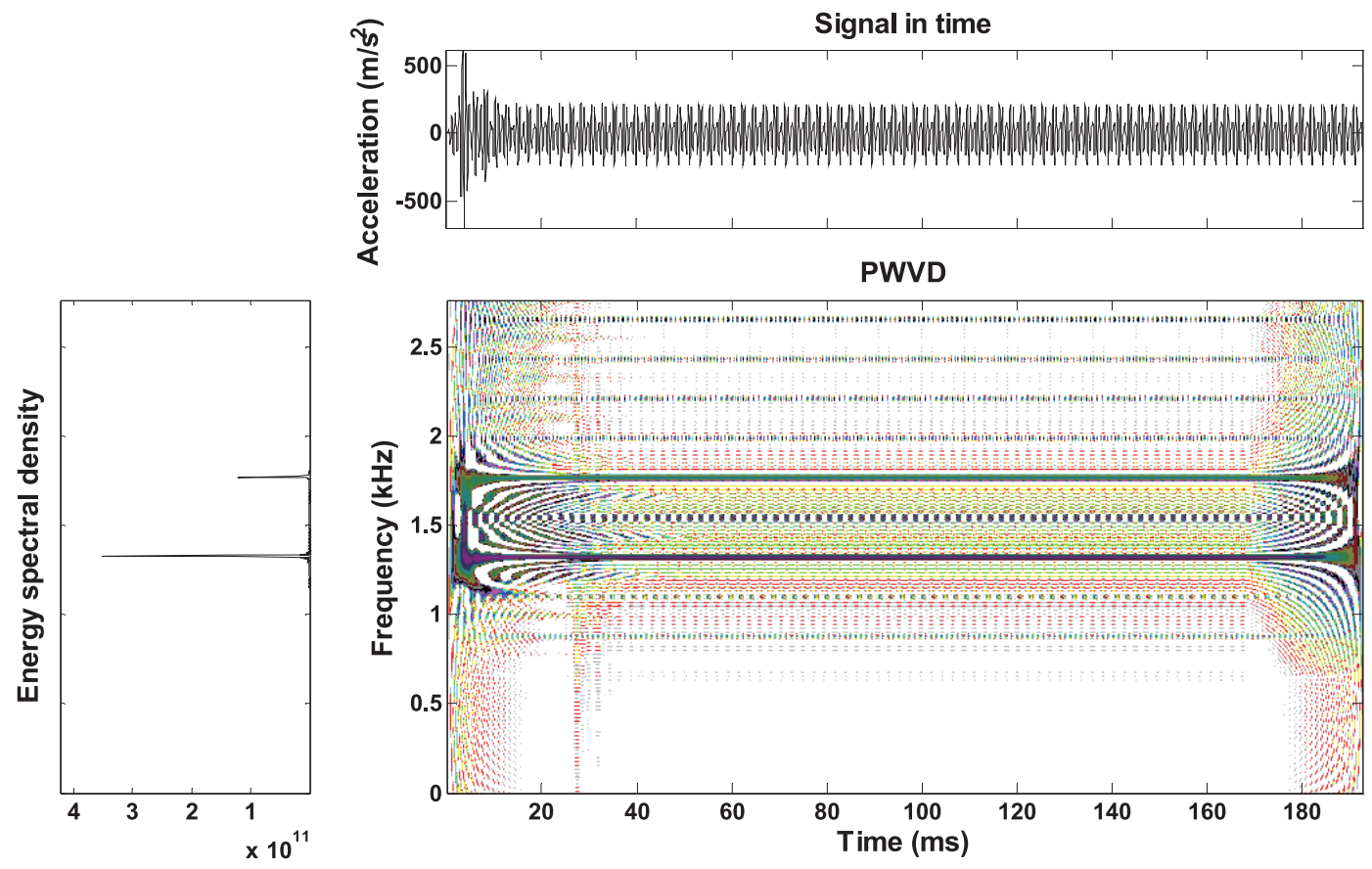

Fig. 13. SWVD of the pinion bearing acceleration for a constant load.
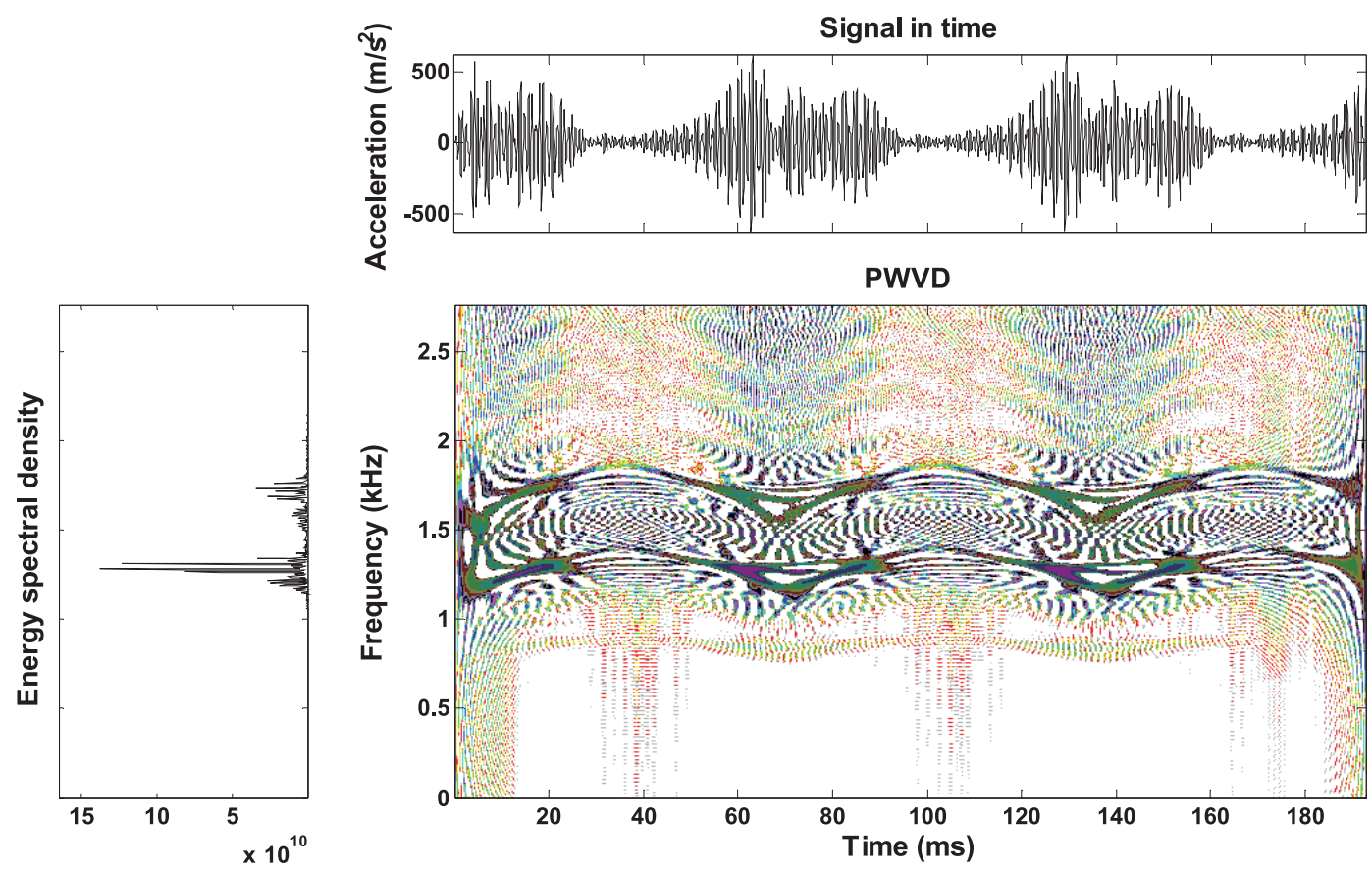

Fig. 14. SWVD of the pinion bearing acceleration for a time varying load. 


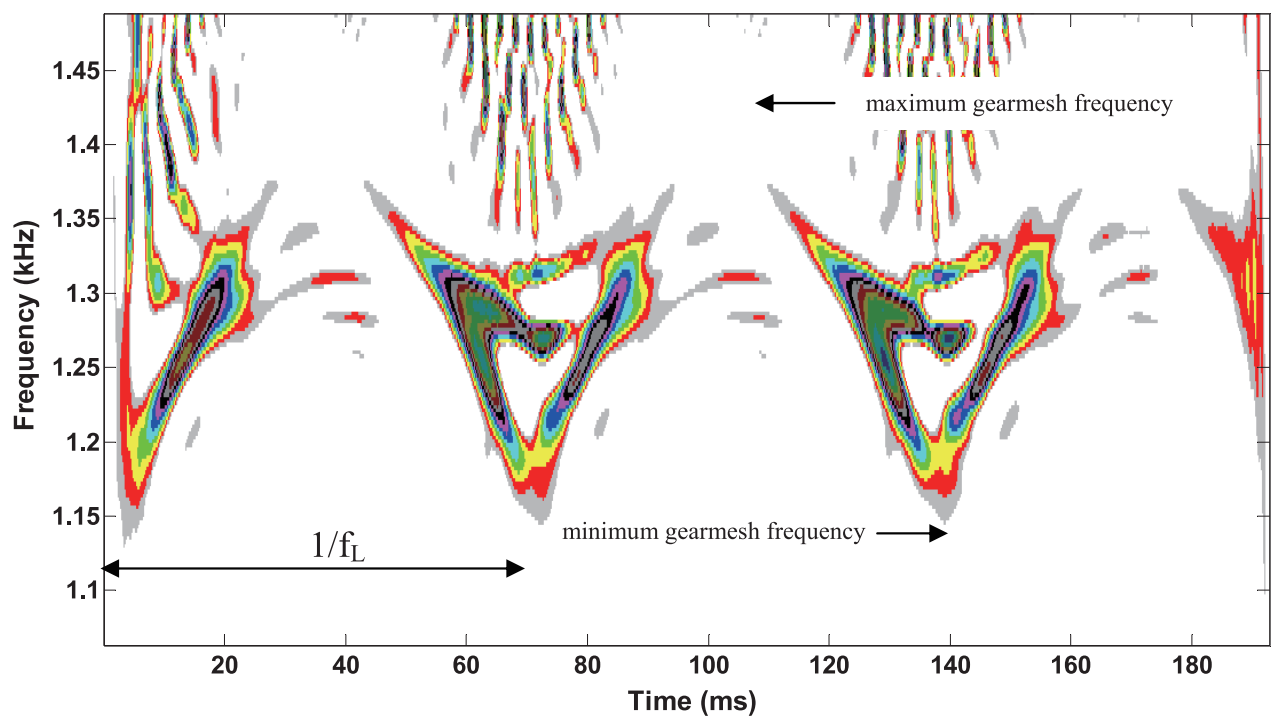

Fig. 15. Details of the SWVD around $3 \times f_{g m}$ for a time varying load.

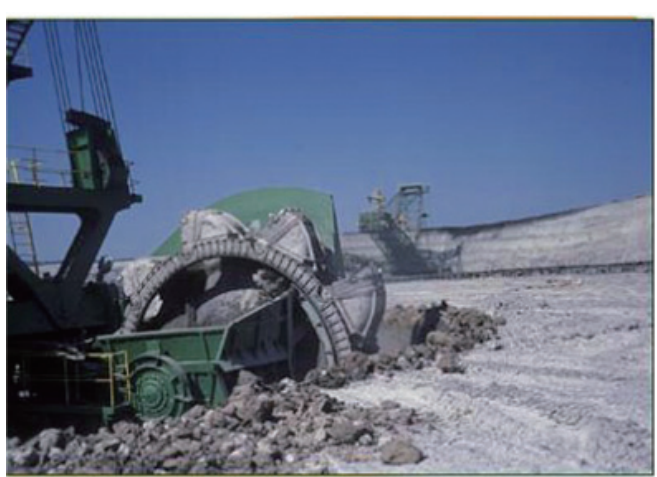

(a)

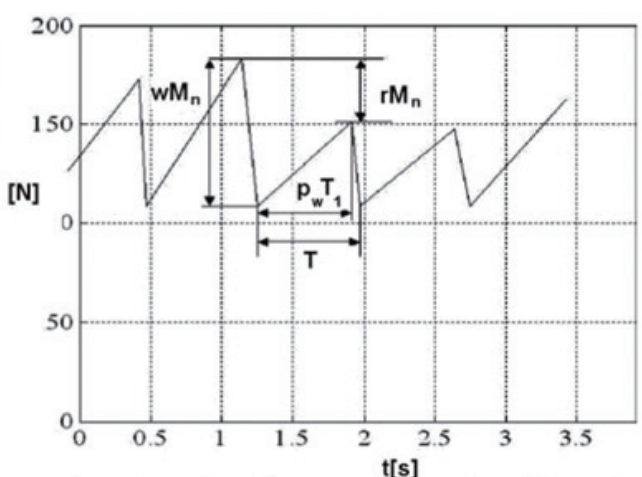

(b)

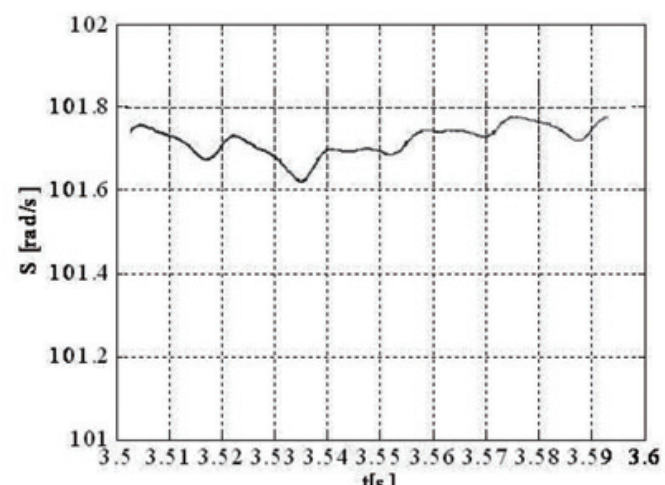

(c)

Fig. 16. (a) View of bucket wheel during operation (b) model of external load variation (c) rotational speed variation caused by varying cyclic load. 


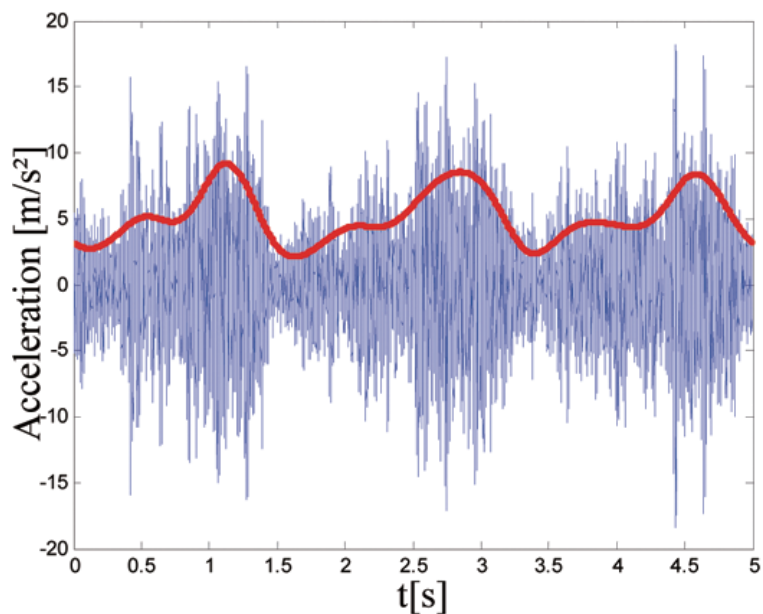

(a)

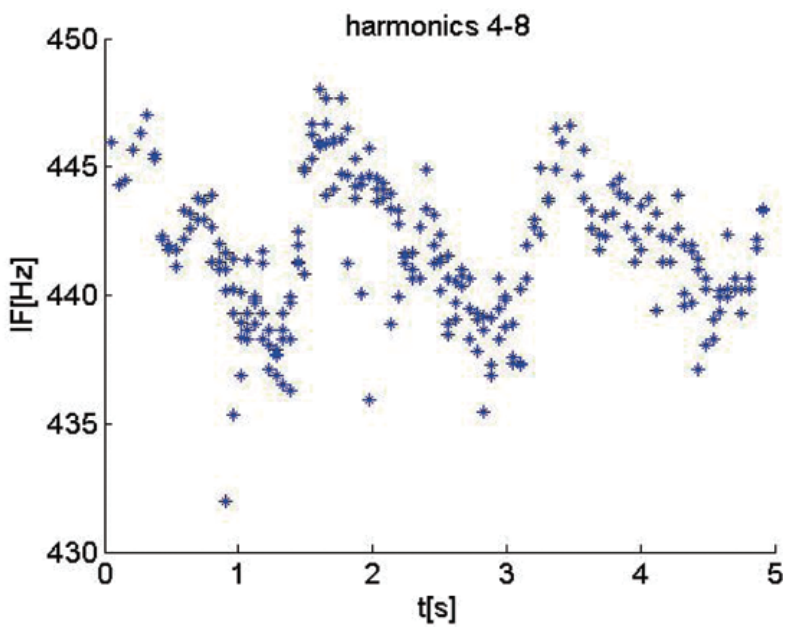

(b)

Fig. 17. Example of load dependent vibration signal: a) cyclic variation of one of mesh harmonic (raw vibration band pass filtered around ith mesh), b) estimated instantaneous mesh frequency profile (results of normalised frequencies for harmonics no 4-8).

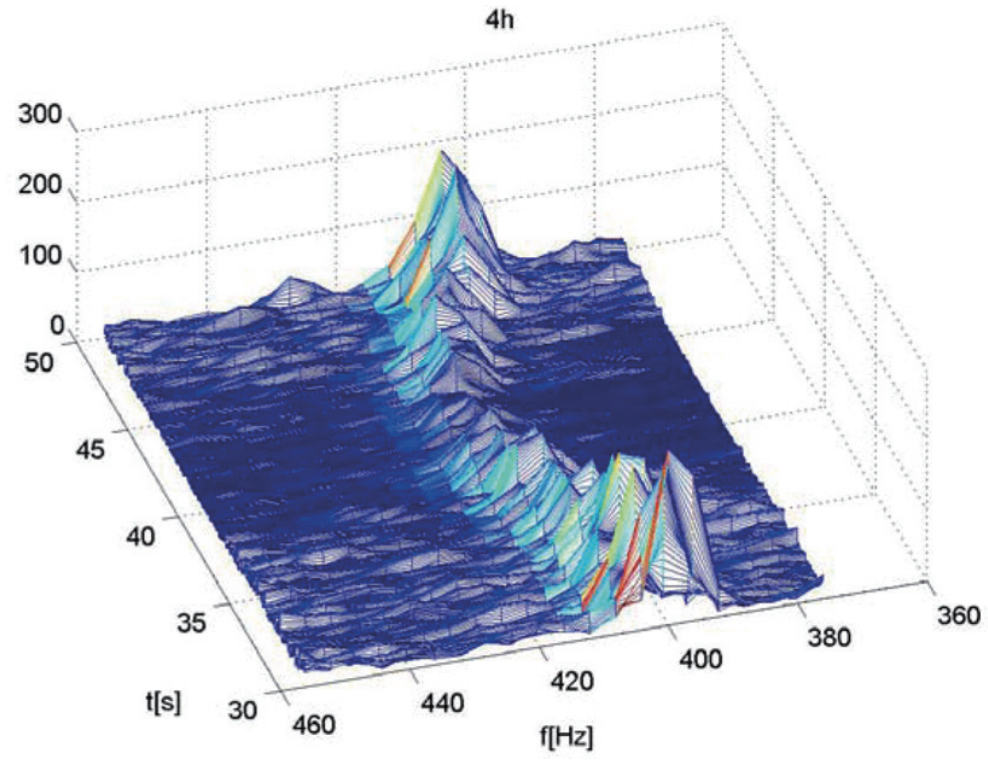

Fig. 18. Example of simultaneous amplitude and frequency variation - spectrogram of pre-filtered signal around mesh harmonic [21].

In order to explain the phenomena existing in such situation a comprehensive mathematical model of a mechanical system with a spur gear transmission under time varying load is presented. It has been shown that this load variation causes a speed variation of the driving motor following a given characteristic and finally it significantly modifies properties of diagnostic signal that can be misleading during condition monitoring/fault detection. In order to get some knowledge about this modification a model based experiment has been used.

To model these phenomena i.e. influence of time varying load to gearbox' vibration response in the gearbox system model, a periodic change of the gearmesh frequency is operated following the evolution of the load and then the speed of the driving motor.

Simulations of the dynamic behavior of the system showed high amplitude modulation in the time responses and sidebands around the gearmesh frequency and its harmonics for the spectra. Amplitudes of mesh (and harmonics) 


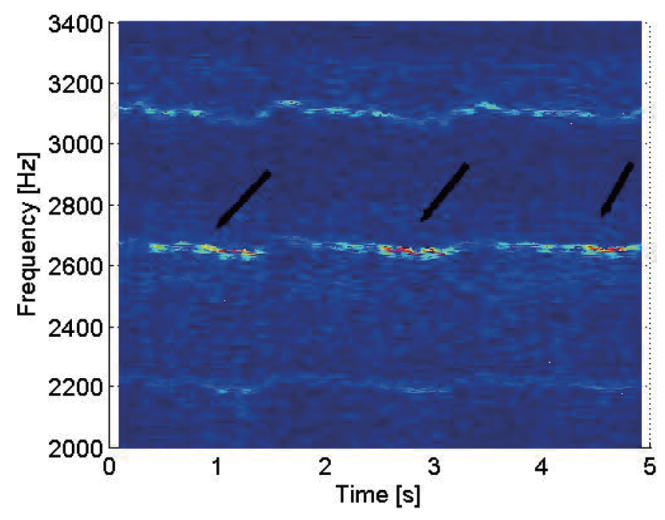

Fig. 19. STFT applied to accelerations measured on the DU bearings.

components are decreasing seriously when load is varying; energy of component is dispersed in some frequency ranges around mesh. Such results may lead to erroneous diagnosis of the transmission, especially in automated systems. To investigate this phenomenon, the joint time frequency smoothed Wigner-Ville distribution showed real performances in the identification of the speed variation induced by the load variation. Experimental results issued from a mining machine confirmed those obtained by numerical simulation. It has been confirmed experimentally, by several techniques discussed in earlier papers that both amplitude and frequency modulation exist in the signal if gearbox works under time varying (cyclic) operating conditions. These modulation make vibration signature much more complex both in time and frequency domains and in some cases diagnostics looks very similar as for damaged gearbox.

Main conclusion from this paper is that, apart from diagnostic features, it is necessary to know information about value and variability of load. In future works the presented model will be a tool for developing relation featuresload-condition of machine for simulated local and distributed faults and also for transient phenomena that appear when heavy loaded machine is rapidly unloaded, especially in the case of over limit bearings backlash.

\section{Acknowledgements}

The Paper is financially supported by Polish State Committee for Scientific Research in 2010-2013 as research project.

\section{References}

[1] W. Bartelmus and R. Zimroz, Vibration condition monitoring of planetary gearbox under random varying, Mechanical Systems and Signal Processing 23 (2009), 246-257.

[2] W. Bartelmus and R. Zimroz, A new feature for monitoring the condition of gearboxes in non-stationary operating conditions, Mechanical Systems and Signal Processing 29 (2010), 637-646.

[3] R.B. Randall, A new method of modelling gear faults, Journal of Mechanical Design 104 (1982), $259-267$.

[4] C.J. Stander, P.S. Heyns and W. Schoombie, Using Vibration Monitoring For Local Fault Detection on Gears Operating Under Fluctuating Load Conditions, Mechanical Systems and Signal Processing 16(6) (2002), 1005-1024.

[5] N. Baydar and A. Ball, Detection of gear deterioration under varying load conditions by using the instantaneous power spectrum, Mechanical Systems and Signal Processing 14 (2000), 907-921.

[6] F. Combet and R. Zimroz, A new method for the estimation of the instantaneous speed relative fluctuation in a vibration signal based on the short time scale transform, Mechanical Systems and Signal Processing 23(4) (2009), 1382-1397.

[7] Y. Zhan, Y. Makis and A.K.S. Jardine, Adaptive State Detection Of Gearboxes Under Varying Load Conditions Based On Parametric Modelling Mechanical, Mechanical Systems and Signal Processing 20(1) (2006), 188-221.

[8] T. Fakhfakh, F. Chaari and M. Haddar, Numerical and experimental analysis of gear system with teeth defects, International Journal of Advanced Manufacturing Technology (IJAMT) 25 (2005), 542-550.

[9] F. Chaari, W. Baccar and M. Haddar, Effect of spalling or tooth breakage on gearmesh stiffness and dynamic response of a one-stage spur gear transmission, European Journal of Mechanics A/Solids 27(4) (2008), 691-705. 
[10] F. Chaari, T. Fakhfakh and M. Haddar, Analytical modelling of spur gear tooth crack and influence on gearmesh stiffness, European Journal of Mechanics - A/Solids 28 (2009), 461-468.

[11] W. Bartelmus, Mathematical modelling of gearbox vibration for fault diagnosis, International Journal of COMADEM 34) (2000), 5-15.

[12] W. Bartelmus, Mathematical modelling and computer simulations as an aid to gearbox diagnostics, Mechanical Systems and Signal Processing 15(5) (2001), 855-871.

[13] D. Wright, Class notes on design and analysis of machine elements, Department of Mechanical and Materials Engineering, The University of Western Australia (2005).

[14] R.G. Parker, S.M. Vijayakar and T. Imago, Non-linear dynamic response of a spur gear pair: modelling and experimental comparisons, Journal of Sound and Vibration 237(3) (2000), 435-455.

[15] O. Sadettin, A. Nizami and C. Veli, Vibration monitoring for defect diagnosis of rolling element bearings as a predictive maintenance tool, Comprehensive case studies, NDT\&E International 39 (2006), 293-298.

[16] Q. Shie and C. Dapang, Joint Time-Frequency Analysis: Method and Application, Prentice Hall; 1st ed., (May 13, 1996).

[17] Y.S. Shin and J.J. Jeon, Pseudo Wigner-Ville Time Frequency Distribution and Its Application to Machinery Condition Monitoring, Journal of Shock and Vibration 1(1) (1993), 65-76.

[18] R. Zimroz and W. Bartelmus, Application of adaptive filtering for weak impulsive signal recovery for bearings local damage detection in complex mining mechanical systems working under condition of varying load, Solid State Phenomena 180 (2012), $250-257$.

[19] W. Bartelmus, F. Chaari, R. Zimroz and M. Haddar, Modelling of gearbox dynamics under time-varying nonstationary load for distributed fault detection and diagnosis, European Journal of Mechanics. A, Solids 29(4) (2010), 637-646.

[20] R. Zimroz, Adaptive approaches for condition monitoring of mining machines, Gospodarka Surowcami Mineralnymi (Mineral Resources Management) 4/3(24) (2008), 103-115.

[21] R. Zimroz, F. Millioz and M. Martin, A procedure of vibration analysis from planetary gearbox under non-stationary cyclic operations by instantaneous frequency estimation in time-frequency domain, Proceedings of Condition Monitoring Conference, Stratford upon Avon, June 2010, UK. 

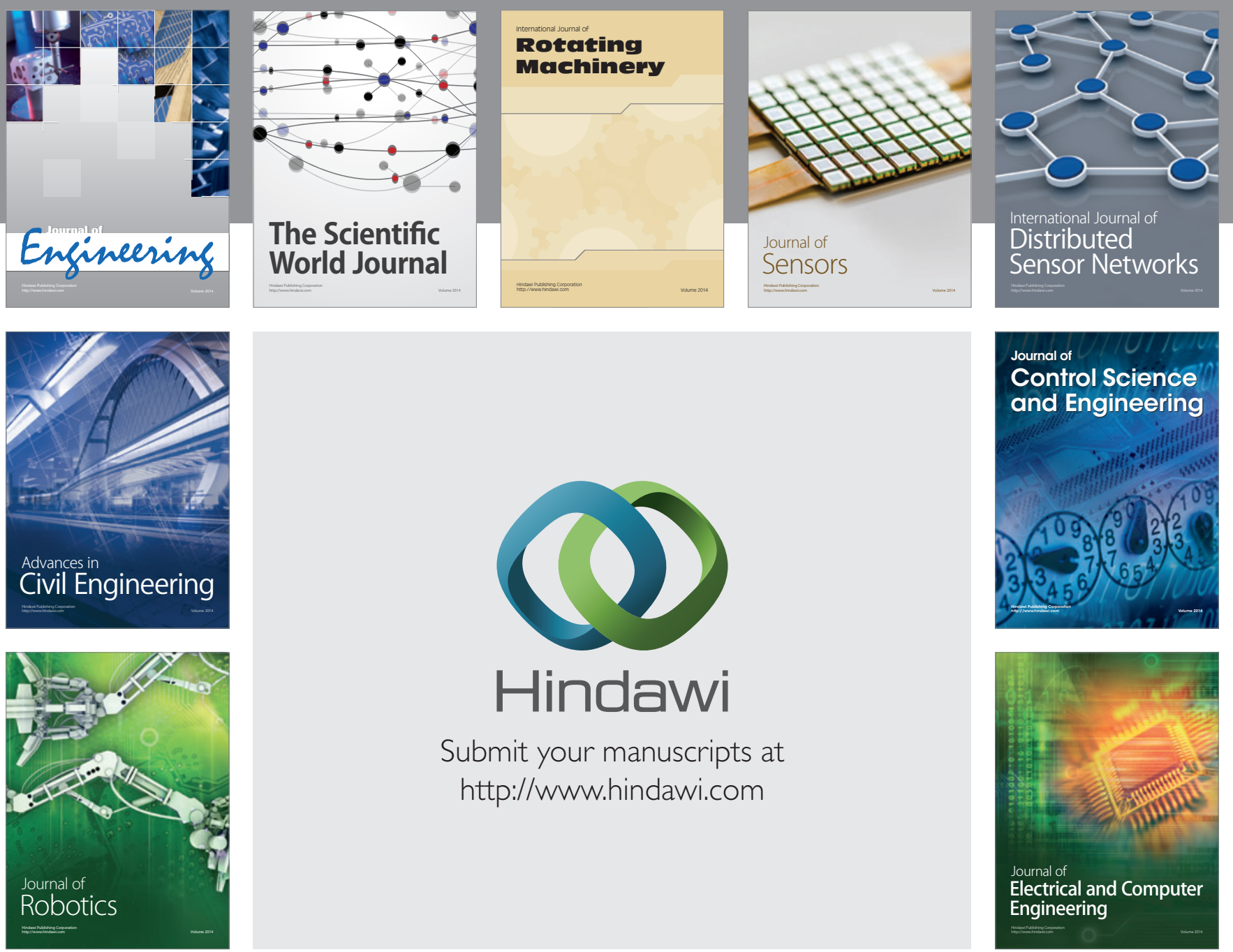

Submit your manuscripts at

http://www.hindawi.com
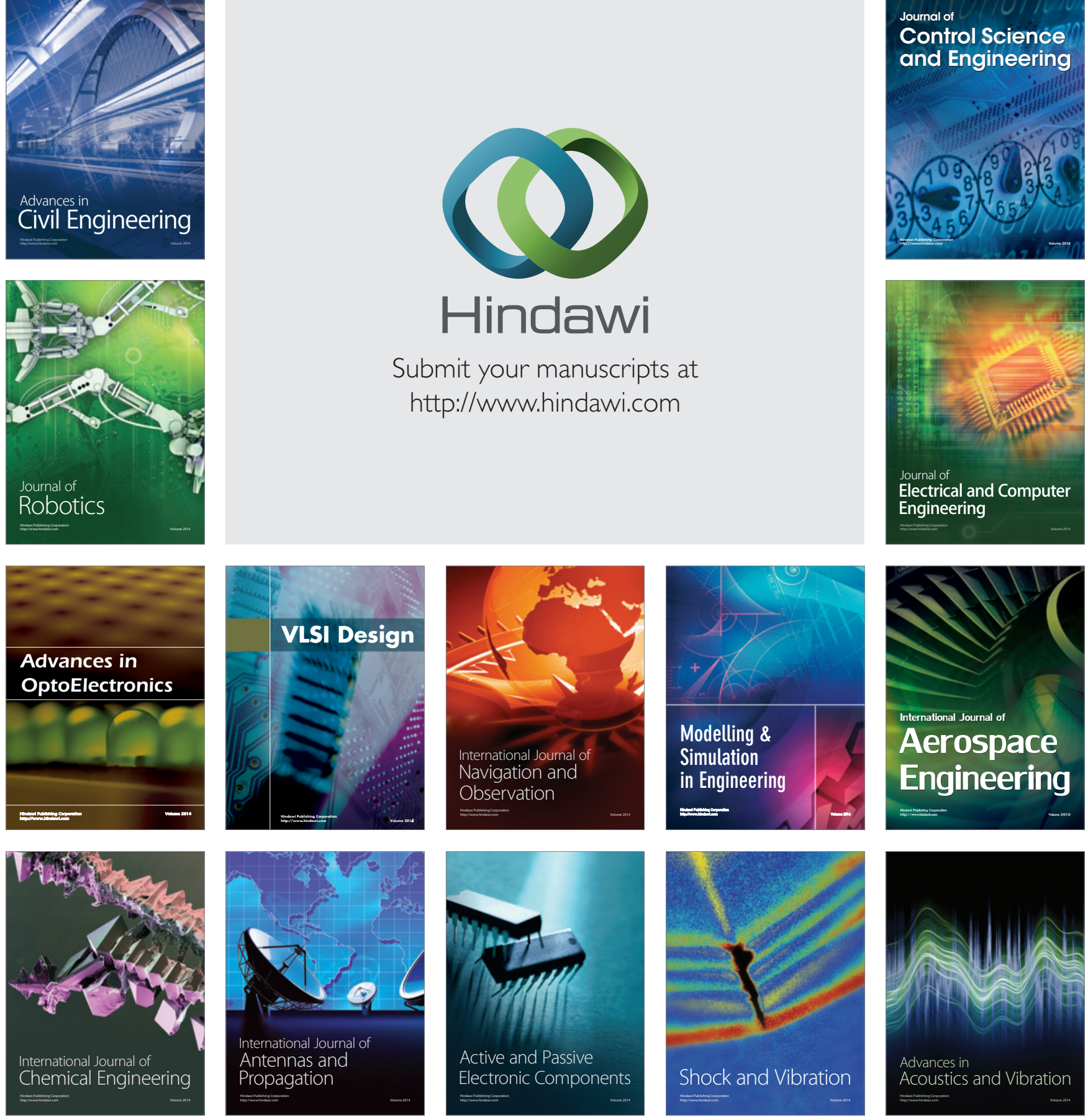